\title{
1 A peroxide-responding sRNA evolved from a peroxidase mRNA
}

3 Madeline C. Krieger ${ }^{\mathrm{a}, 1}$, H. Auguste Dutcher ${ }^{\mathrm{a}, 1}$, Andrew J. Ashford ${ }^{\mathrm{a}}$, Rahul Raghavan ${ }^{\mathrm{a}, \mathrm{b}, 2}$

$5 \quad$ a Department of Biology, Portland State University, Portland, OR 97201, USA.

6 bepartment of Molecular Microbiology and Immunology, The University of Texas at San

7 Antonio, San Antonio, TX 78249, USA.

8

$9 \quad{ }^{1} \mathrm{MCK}$ and $\mathrm{HAD}$ contributed equally to this work.

$11{ }^{2}$ Author for correspondence:

12 Rahul Raghavan | Phone: 210-458-7016 | Email: rahul.raghavan@utsa.edu

13

14 Key words: OxyS, sRNA, sRNA evolution, peroxidase, peroxide 


\section{ABSTRACT}

17 Small RNAs (sRNAs) are critical regulators of gene expression in bacteria, but we lack a clear

18 understanding of how new sRNAs originate and get integrated into regulatory networks. A

19 major obstacle to elucidating their evolution is the difficulty in tracing sRNAs across large

20 phylogenetic distances. To overcome this roadblock, we investigated the prevalence of sRAs in

21 more than a thousand genomes across Enterobacterales, a bacterial order with a rare confluence

22 of factors that allows robust genome-scale sRNA analyses: several well-studied organisms with

23 fairly conserved genome structures, an established phylogeny, and substantial nucleotide diversity

24 within a narrow evolutionary space. Using a covariance modeling-based approach, we analyzed

25 the presence of hundreds of sRNAs and discovered that a majority of sRNAs arose recently, and

26 uncovered protein-coding genes as a potential source for the generation of new sRNA genes. A

27 detailed investigation of the emergence of OxyS, a peroxide-responding sRNA, demonstrated

28 that it evolved from a $3^{\prime}$ end fragment of a peroxidase mRNA. Collectively, our data show that

29 the erosion of protein-coding genes can result in the formation of new sRNAs that continue to

30 be part of the original protein's regulon. This novel insight provides a fresh framework for

31 understanding how new sRNAs originate and get incorporated into preexisting regulatory

32 networks. 


\section{AUTHOR SUMMARY}

35 Small RNAs (sRNAs) are important gene regulators in bacteria, but it is unclear how new

36 sRNAs originate and become part of regulatory networks that coordinate bacterial response to

37 environmental stimuli. Here, we show that new sRNAs could arise from protein-coding genes

38 and potentially be incorporated into the ancestral proteins' regulatory networks. We illustrate

39 this process by defining the origin of OxyS. This peroxide-responding sRNA evolved from and

40 replaced a peroxidase gene, but continues to be part of the peroxide-response regulon. In sum,

41 we describe the source from which OxyS, one of the most well-studied sRNAs, arose, identify

42 protein-coding genes as a potential raw material from which new sRNAs could emerge, and

43 suggest a novel evolutionary path through which new sRNAs could get incorporated into pre-

44 existing regulatory networks.

45

46 


\section{INTRODUCTION}

48 Bacterial small RNAs (sRNAs) control gene expression by modulating translation or by altering

49 the stability of messenger RNAs (mRNAs). sRNAs allow precise and efficient control of gene

50 expression because they are produced quickly, regulate multiple genes simultaneously, and could

51 degrade along with target mRNAs (1). These qualities are especially beneficial under conditions

52 such as oxidative stress that require abrupt reprogramming of regulatory networks (2). In

53 bacteria, oxidative stress caused by hydrogen peroxide $\left(\mathrm{H}_{2} \mathrm{O}_{2}\right)$ is mitigated mainly by peroxidases

$54(3,4)$. For instance, a peroxidase system encoded by $a h p C F$ genes is induced by the regulator

55 OxyR when Escherichia coli is exposed to $\mathrm{H}_{2} \mathrm{O}_{2} ; \mathrm{OxyR}$ simultaneously upregulates the expression

56 of several other genes, including the sRNA OxyS that together assuage $\mathrm{H}_{2} \mathrm{O}_{2}$ toxicity (5-9).

57 OxyS is one of the most well-studied sRNAs. More than two decades of research on this

58 sRNA has revealed many of the foundational details about sRNA-mediated gene regulation (10-

59 15). In E. coli and Salmonella enterica, OxyS is encoded by a gene located in the intergenic region

60 (IGR) between $\operatorname{xy} R$ and $\arg H$ genes. Similar to OxyS, most sRNAs in bacteria are transcribed

61 from genes present in IGRs; however, in recent years, numerous sRNAs that are encoded within

62 protein-coding genes have also been identified. In such cases, sRNAs are either transcribed from

63 promoters contained within protein-coding sequences or are generated from mRNAs by

64 endoribonucleases that act in concert with the chaperone protein Hfq (17-22). sRNAs originate in bacteria (23). One of the main impediments to elucidating the evolutionary

67 histories of sRNAs is the difficulty in tracing sRNAs across large phylogenetic distances (24).

68 Unlike proteins that are fairly easy to identify in distant bacteria, sRNAs can only be reliably 
70 factors, including their small size (50 to $400 \mathrm{nt}$ ), rapid turnover, and a lack of open reading

71 frames (ORFs) or other features that serve as signposts (23-27). Given these constraints, an ideal

72 group of bacteria to study sRNA evolution is the order Enterobacterales (25), which has an

73 established phylogeny, substantial nucleotide diversity within a narrow evolutionary space, and

74 contains well-characterized organisms with diverse lifestyles but enough similarity in genome

75 structure to enable meaningful comparative genomics.

76 Here, by analyzing the prevalence of hundreds of sRNAs in more than a thousand

77 Enterobacterales genomes, we show that most sRNAs arose recently, and that mRNAs are a

78 potential source for the generation of new sRNAs. One sRNA that originated from an mRNA is

79 OxyS, which evolved from a 3' end fragment of a peroxidase mRNA. Interestingly, both the

80 parental peroxidase and OxyS are regulated by OxyR, suggesting a novel paradigm for

81 understanding how new sRNAs arise and are recruited into preexisting regulatory networks:

82 Erosion of a protein-coding gene could lead to the formation of a new sRNA gene that

83 continues to function as part of the original protein's regulatory network.

85 RESULTS

86 Most sRNAs in enteric bacteria arose recently

87 We built covariance models for 371 sRNAs described in E. coli K-12 MG1655, S. enterica

88 Typhimurium SL1344, and Yersinia pseudotuberculosis IP32953, and located their homologs

89 across 1105 Enterobacterales genomes. The ensuing phyletic patterns of sRNA presence and

90 absence was used to perform an evolutionary reconstruction of ancestral states using a maximum

91 likelihood approach (Figure 1, Table S1). This order-wide analysis showed that 61\% of sRNAs 
92 (228/371) emerged at the root of a genus or more recently (categorized as “young”). In

93 comparison, among 148 proteins that function as gene regulators in E. coli and S. enterica, only

$9418 \%$ fall in this category (Figure S1, Table S2). The overrepresentation of recently-evolved

95 sRNAs in our dataset indicates that sRNAs arise rapidly, probably in response to lineage-specific

96 selection pressures. It should be noted however that the functions, if any, of most recently-

97 emerged sRNAs have not been determined, and that nearly all sRNAs with known functions

98 have putative origins ancestral to the root of their respective genera (belong to the "middle" and

99 “old” categories) (Table S1).

100

101 Protein-coding genes are potential progenitors of sRNA genes

102 Our covariance modeling-based search identified 62 sRNAs that were located in IGRs in the

103 hub genomes (E. coli K-12 MG1655, S. enterica Typhimurium SL1344, or Y. pseudotuberculosis

104 IP32953) but mapped to the coding strands of protein-coding genes in other Enterobacterales

105 members (Table S3). A majority of the overlaps were at the $3^{\prime}$ ends of genes (34/62), while 18

106 were at $5^{\prime}$ ends and 10 within gene boundaries. The sRNA-ORF overlaps suggest that some of

107 the sRNAs were originally part of mRNAs, and later evolved into independent sRNAs when the

108 protein-coding genes decayed, leaving behind only the sRNA-encoding segments.

To better understand their evolutionary histories, we examined several sRNAs that

110 overlapped protein-coding genes with annotated functions in more detail. This analysis revealed

111 that OxyS, an sRNA produced in response to peroxide stress in E. coli and S. enterica, overlapped

112 the $3^{\prime}$ end of a peroxidase gene in Serratia (Figure 2). The peroxidase gene is located in the same

113 genetic context - divergent from $\operatorname{oxy} R$, as OxyS is in E. coli and S. enterica, denoting that the 
114 sRNA likely evolved from the peroxidase gene. In addition, the promoter regions of both oxyS

115 and peroxidase genes contain OxyR-binding sites, indicating that the expression of the

116 peroxidase gene is controlled by OxyR, as shown for OxyS $(6,10)$.

Another sRNA that seems to be part of its parental protein's regulatory circuit is StyR-3.

118 This sRNA of unknown function is highly abundant in S. enterica (28). It shares sequence

119 homology with the $5^{\prime}$ end of an MBL-fold metallohydrolase (MMH) gene in Klebsiella, and

120 both StyR-3 and MMH are located divergently from the transcriptional regulator gene $\operatorname{ramR}$. In

121 addition, the IGR between $r a m R$ and StyR-3/MMH contains a RamR-binding site (Figure 2).

122 The sequence similarity, homologous genetic location, and conservation of RamR-binding site

123 suggest that StyR-3 evolved from the $5^{\prime}$ end of the MMH gene and continues to be under the

124 regulatory control of the divergently encoded RamR.

125 A third example of an sRNA that appears to have evolved from a degraded protein-

126 coding gene is STnc240, an sRNA with unknown function in S. enterica. The gene for this

127 sRNA is located between yoeI and yeeY genes in Salmonella species, but in Cronobacter, the yoeI-

128 yeeYIGR contains a 4-aminobutyrate-2-oxoglutarate transaminase (gabT) gene whose $3^{\prime}$ end

129 contains a sequence that is very similar to that of STnc240 (Figure 2). Transcriptional regulation

130 of gabT and STnc240 are not well-defined, but sequence homology and conservation of genetic

131 location suggest that the sRNA arose from the remnants of the gabT gene.

133 is STnc3230. This “young” sRNA likely emerged from the 3' end of a 1,3-1,4-beta-glucanase

134 sugar-binding protein (SBP) (Figure 2). While both $S$. bongori and S. enterica Arizonae contain a 135 gene for SBP between $d a p B$ and carA genes, STnc3230, which shares sequence similarity with 3' 
136

137

138

140

141

142

143

144

145

146

147

148

149

150

151

152

153

154

155

156

157

end of the SBP gene, is located in this IGR in S. enterica Typhimurium. Interestingly, DapZ, an sRNA transcribed from the 3' end of $d a p B$ gene is also located in the same IGR as STnc3230, but on the opposite DNA strand (21).

Lastly, an sRNA that seem to have evolved from within a protein-coding gene is IsrK. This prophage-encoded sRNA likely originated from the ASH domain of a bacteriophage protein-coding gene (29), and evolved to regulate the expression of a prophage-encoded antiterminator protein AntQ(30). Similar to the origin of IsrK from a degenerated prophage gene, we have shown previously that EcsR2, an sRNA present in E. coli, evolved from a degraded phage tail fiber gene (26). Additionally, three sRNAs (Esr2, Esr4 and Ysr232) overlap genes that encode transposases and integrases (Table S3), suggesting that they arose in transposons or insertion sequences, as we showed recently for sRNAs in the pathogen Coxiella burnetii (31). Of these eight sRNAs, we focus on the origin of OxyS in the rest of this article.

\section{A peroxidase gene was replaced by oxy $S$ gene in Enterobacteriaceae}

In the order Enterobacterales, oxy $S$ gene is present only in the family Enterobacteriaceae (e.g., $E$. coli, S. enterica), where it is located divergently from the oxyR gene in the oxyR-argH IGR

(Figure 3). In contrast, a peroxidase (peroxiredoxin-glutaredoxin hybrid) gene occupies the same locus in families Erwiniaceae, Pectobacteriaceae, Yersiniaceae, Hafniaceae, and Budviciaceae. Bacteria belonging to orders Pasteurellales and Vibrionales also contain orthologous peroxidase genes at this location (Figure 3). The most parsimonious explanation for this phylogenetic profile is that the peroxidase gene was present in the common ancestor of all Enterobacterales and that it was subsequently replaced by the oxyS gene in Enterobacteriaceae. In addition, a 
158 closer analysis of OxyS-peroxidase sequence overlap showed that the last $~ 55$ nt of the peroxidase

159 coding sequence, $25 \mathrm{nt}$ of the $3^{\prime}$ UTR and the intrinsic terminator likely transformed into OxyS

160 (Figure 4). Based on these data, we conclude that oxyS gene present in Enterobacteriaceae is the

161 remnant of the $3^{\prime}$ end of the ancestral peroxidase gene present in the rest of the members of the

162 order Enterobacterales.

164 Exposure to $\mathrm{H}_{2} \mathrm{O}_{2}$ induced peroxidase expression and production of mRNA fragments

165 Similar to $\operatorname{xyy} S$, the peroxidase gene is located divergently from oxyR and the IGR between the 166 two genes contain putative OxyR-binding sites (Figure 3, Figure S2). To test whether the

167 expression of the peroxidase gene is induced by $\mathrm{H}_{2} \mathrm{O}_{2}$, we exposed Serratia marcescens (family

168 Yersiniaceae), Edwardsiella hoshinae (family Hafniaceae), and a representative from outside of

169 Enterobacterales: Vibrio harveyi (order Vibrionales, family Vibrionaceae) to $1 \mathrm{mM}$ of $\mathrm{H}_{2} \mathrm{O}_{2}$ for 10

170 minutes. As observed for OxyS in E. coli (10), exposure to $\mathrm{H}_{2} \mathrm{O}_{2}$ significantly induced the

171 expression of the peroxidase gene in all three bacteria (Figure S3), indicating that it is also

172 regulated by OxyR. Additionally, peroxidase mRNAs in all three bacteria produced small 3'

173 fragments that correspond to the region from where OxyS emerged (Figure 5). Although the

174 size of the cleavage products are not identical in the three bacteria, the fragmentation process

175 itself appears to be a trait ancestral to all Enterobacterales, thus providing the raw material from

176 which OxyS eventually evolved in an Enterobacteriaceae ancestor. 


\section{DISCUSSION}

181 Despite their importance to bacterial physiology and virulence, the evolutionary processes that

182 produce new sRNAs are not well understood. One of the main reasons for this lack of clarity

183 about sRNA origination is that unlike protein-coding genes, sRNA genes are difficult to trace

184 across large phylogenetic distances (24). Following up on previous research that showed that

185 enteric bacteria are at optimum distances from one another to effectively investigate sRNA

186 prevalence (25), we traced the presence of hundreds of sRNAs across Enterobacterales and show

187 that a majority emerged recently in a lineage-specific manner. This observation fits with earlier

188 findings that showed that sRNAs evolve rapidly in bacteria and are typically genus- or species-

189 specific $(26,32-35)$. Interestingly, we found that most well-studied sRNAs belong to "middle"

190 and "old" age groups. A similar observation was made by a study that examined the evolutionary

191 histories of 58 experimentally validated sRNAs in E. coli (36). Although specific age categories

192 were not assigned in that study, when we classified the sRNAs into three age groups based on

193 the distance of gain-node from $E$. coli (old: >0.1; middle: 0.001 - 0.009; young: 0.0001 - 0.0009),

$19451 / 58$ sRNAs were deemed to be old or middle-aged (Table S4). This biased representation is

195 probably due to the propensity of older sRNAs to be expressed at high levels, thereby making

196 them more amenable to discovery and experimental validation $(26,37)$.

197

We have previously shown that new sRNAs arise de novo and from degraded

198 bacteriophage- and transposon-associated genes $(23,26,31,33)$. In this study, we report that

199 protein-coding genes could serve as a raw material for sRNA biogenesis and support this

200 conclusion by showing that OxyS, a peroxide-responding sRNA, originated from a peroxidase

201 gene. OxyS was first noticed by researchers because it is transcribed divergently from the oxyR

202 gene that encodes a transcriptional regulator that orchestrates E. colis antioxidant response (10). 
203 The IGR between oxyS and $\operatorname{xyy} R$ genes contains OxyR-binding sites from where the oxidized

204 form of OxyR induces the expression of OxyS in response to $\mathrm{H}_{2} \mathrm{O}_{2}$ exposure (10,38-40). We

205 show that the $\operatorname{xxy} R$-oxyS gene arrangement is present only in the family Enterobacteriaceae,

206 whereas a peroxidase gene, whose expression is also induced by $\mathrm{H}_{2} \mathrm{O}_{2}$, occupies the genetic locus

207 next to $\operatorname{oxy} R$ in other members of the order Enterobacterales. The evolutionary process that led

208 to the replacement of the ancestral peroxidase gene by oxyS gene could have occurred through

209 two possible routes (Figure 6). In one, the mRNA 3' end fragment gained a regulatory function,

210 which resulted in its retention when the rest of the peroxidase gene was pseudogenized and later

211 deleted in Enterobacteriaceae. Alternatively, the peroxidase gene was pseudogenized first, but

212 continued to be transcribed producing the $3^{\prime}$ end fragment, which gained a regulatory function

213 and was retained when the rest of the gene was deleted.

214 An unresolved question in the field of sRNA biology is how new sRNAs become

215 incorporated into regulatory networks. This study provides a possible explanation: An sRNA

216 arising from a protein-coding gene would inherently be part of the parental protein's regulon.

217 For instance, in either scenario in Figure 6, the mRNA fragment that gave rise to OxyS would

218 have been produced as part of the OxyR regulon even before it gained any function. Thus, when

219 functional OxyS later emerged from that fragment, it was already part of the OxyR regulatory

220 network. Similar to the origin of OxyS, 3' ends of mRNAs appear to be the most favorable

221 location for sRNA genesis (34/62 in our dataset) probably due to the presence of intrinsic

222 terminators that improve RNA stability and promote Hfq binding, two factors that are critical to

223 sRNA evolution and function (27, 41-44). The next best seem to be $5^{\prime}$ ends $(18 / 62)$, likely due

224 to proximity to promoter regions that regulate transcription, with middle region being the least 
225 likely (10/62) to contribute to sRNA evolution. Irrespective of this apparent difference, any part

226 of an mRNA could potentially evolve into a regulatory RNA, as shown in a recent study that

227 demonstrated the generation of sRNA-like transcripts from 5', middle and $3^{\prime}$ segments of

228 mRNAs in E. coli (20). In sum, because bacterial genomes contain numerous protein-coding

229 genes in various stages of decay $(45,46)$, they could function as a rich resource from which new

230 sRNAs could arise rapidly in response to lineage-specific environmental pressures.

\section{MATERIALS AND METHODS}

233 Determining sRNA presence across the order Enterobacterales

234 A list of candidate-sRNAs in E. coli K-12 MG1655 (NC_000913.3), S. enterica Typhimurium

235 SL1344 (NC_016810.1), and Yersinia pseudotuberculosis IP32953 (NC_006155.1) were compiled

236 from previously published studies $(37,40,47)$. Several exclusion criteria were used to remove

237 spurious and redundant sRNAs: (i) sRNAs under 60 nucleotides in length, (ii) sRNAs that

238 overlapped each other by more than 10 nucleotides, (iii) sRNAs that were present in multiple

239 copies, (iv) RNAs that were identified as cis-acting in the Rfam database (48), and (v) sRNAs

240 that shared $95 \%$ or more nucleotide identity over at least 60 nucleotides of their lengths. For

241 each sRNA of interest, a representative sequence from each hub genome was used as the query

242 for a BLASTn (wordsize 7, maxdbsize $100 \mathrm{~Kb}$, dbsize normalized to $4 \mathrm{Mb}$, evalue $\leq 1 \mathrm{e}-5$ ) using

243 BLAST v2.7.1 against a database of 1105 Enterobacterales genomes (Table S5; Figure S4) that

244 met the following criteria: (i) full genome sequence was available on GenBank, and (ii) the

245 genome was within 0.08 16S rDNA pairwise distance from the hub species (Table S6). Drawing

246 on guidance from previous studies $(24,25)$, hits with pident $>65 \%$ covering at least $95 \%$ of the 
247 length of the original query served as seed sequences from which to construct a covariance model.

248 Candidate hits were next binned by percent identity, and a randomly selected set of sequences

249 (one from each percent identity bin) were chosen to serve as a seed sequence for the covariance

250 model. These sequences were aligned using ClustalW, and the Infernal suite of tools (v1.1.2) was

251 used for subsequent covariance model construction (cmbuild), calibration (cmcalibrate), and

252 homolog searches (cmsearch) (49). Models were constructed from the BLAST-derived seed

253 sequences using cmbuild, while cmscan was used to identify sRNAs already represented by

254 existing Rfam models. These newly constructed models, plus the existing Rfam models, were

255 then used in parallel to search the 1105-genome database for homologs. For sRNAs that were

256 represented by an existing Rfam model, cmsearch results from this model were compared to that

257 from the newly constructed model, and the model that yielded more hits was selected for

258 continued iteration. Results from cmsearch with an e-value $<1 \mathrm{e}-5$ were used to add

259 unrepresented sequences to the query model, which was then refined, recalibrated, and used for

260 another round of cmsearch. This process was repeated for each sRNA until a cmsearch with its

261 corresponding model failed to yield new unrepresented sequences. In order to ensure that any

262 two models were not yielding the same set of hits, results from cmsearch with the finalized

263 models were compared across sRNAs; models with redundant hits were omitted, as were any

264 models that yielded $>1 \mathrm{e} 4$ hits. An sRNA gene was considered present in a given organism if a hit

265 of e-value $<1$ e-5 was found on its chromosome and/or plasmid. All resultant hits were cross-

266 checked by genome location to ensure that a given hit was not represented more than once in the

267 final results. Presence/absence data for all 1105 organisms was collected, but only data for 89

268 Enterobacteriaceae, plus Vibrio cholerae E1 Tor str. N16961 as an outgroup, was used for

269 downstream phylogenetic analyses. 


\section{Evolutionary reconstruction}

272 Enterobacterales phylogenetic tree was downloaded from MicrobesOnLine, and node of origin

273 for each sRNA was determined using the Gain and Loss Mapping Engine (GLOOME) as

274 described previously $(36,50)$. For sRNAs present in a single hub genome (E. coli K-12 MG1655,

275 S. enterica Typhimurium SL1344 or Y. pseudotuberculosis IP32953), the determined gain node was

276 the most ancestral node with a posterior probability of $\geqslant 0.6$, where all nodes leading from this

277 ancestor to the hub genome had a posterior probability $\geqslant 0.6$. If an sRNA was present in more

278 than one hub genome, and the most recent last common ancestor (LCA) of the hub genomes in

279 which it was present had a posterior probability $\geqslant 0.6$, the gain node was the most ancestral node

280 with a posterior probability $\geqslant 0.6$, where all nodes leading from this ancestor to the

281 aforementioned LCA had a posterior probability $\geqslant 0.6$. sRNAs that emerged at the root of a

282 genus or more recently were classified as "young" ( $n=228)$, those present at the last common

283 ancestor of all three hub genomes were deemed to be "old" ( $\mathrm{n}=57)$, and sRNAs that emerged in

284 between the two age groups were considered "middle" ( $n=73)$. If the last common ancestor of the

285 hub genomes in which the sRNA was present did not have a posterior probability of $\geq 0.6$, ages

286 of these sRNAs were considered undetermined $(n=13)$.

287

288 Regulatory protein tree

289 Using the QuickGO annotation table (51), GO:0010629 (negative regulation of gene

290 expression) and its child terms were utilized to identify regulatory proteins in S. enterica

291 Typhimurium LT2 and Escherichia coli K-12 MG1655. Protein sequences (UP00000104, 
292 UP000000625) were downloaded from Uniport (52). Redundancies among the regulatory

293 proteins from the two different species were identified using a BLASTp of the regulatory protein

294 sequence set against itself (pident $>80$, e-value $<1 \mathrm{e}-10$ ). Homologs of this final set of query

295 proteins were identified using BLASTp (e-value <1e-10) against a database of protein sequences

296 from the genomes used for phylogenetic analysis, yielding a presence/absence matrix. Node of

297 origin was determined using GLOOME as described above.

298

299 Finding oxyR-argH IGRs

300 To find $\operatorname{oxy} R$ and $\arg H$ orthologs, tBLASTn searches were carried out with OxyR and ArgH

301 sequences from E. coli K-12 MG1655 against Enterobacterales genomes (e-value $\leq 10 \mathrm{e}-10$,

302 percent positive $\geq 60 \%$, percentage alignment length $\geq 60 \%$ ). We corroborated the tBLASTn hits

303 by confirming that they contain Pfam domains PF03466.20 and PF00126.27 (for OxyR), and

304 PF00206.20 and PF14698.6 (for ArgH) (53). We then compiled a list of bacteria that have both

$305 \operatorname{axy} R$ and $\arg H$ genes and obtained the nucleotide sequences between the two genes using the

306 Entrez E-utilities tool.

307

308 Identifying $5^{\prime}$ neighbors of $o x y R$ and OxyR-binding sites

309 Using BioPython (54), we first determined the direction of the gene next to oxyR's $5^{\prime}$ end. If the

310 neighboring gene was oriented divergently, we determined the identity of the encoded protein

311 using Pfam as described above. All Enterobacterales and Vibrionales (except Morganellaceae)

312 included in this study contained a peroxidase gene with Glutaredoxin (PF00462) and Redoxin

313 (PF08534) domains in this locus. To identify putative OxyR-binding sites located in the IGR 
314 between $\operatorname{oxy} R$ and its neighbor, we extracted 50 bp at the $5^{\prime}$ end of $\operatorname{oxy} R$ along with $100 \mathrm{bp}$ of

315 the adjoining IGR from each bacterium. Sequences were aligned with MUSCLE (55) and the

$31650 \mathrm{bp}$ oxyR sequence was trimmed from each sequence. Using Multiple Em for Motif Elicitation

317 (MEME) (56), we first detected 37 bp palindromic sequences in IGRs, and then used the

318 output from MEME in Find Individual Motif Occurrences (FIMO) (57) to identify putative

319 OxyR-binding site in each bacterium. Sequence logos were generated using WebLogo 3 (58).

320

321 Bacterial growth and gene expression

322 Bacteria were inoculated 1:100 from an overnight culture into fresh media. S. marcescens ATCC

32313880 was grown at $37^{\circ} \mathrm{C}$ in Lysogeny broth (LB), E. hoshinae ATCC 35051 at $26^{\circ} \mathrm{C}$ in LB, and

$324 V$. harveyi ATCC 43516 at $26^{\circ} \mathrm{C}$ in Marine Broth, all shaking at $200 \mathrm{rpm}$. All cultures were

325 grown to an OD600 value of 0.4-0.5, and split into two. One half was allowed to grow under the

326 same conditions for 10 minutes, while the other half was exposed to $1 \mathrm{mM}$ of $\mathrm{H}_{2} \mathrm{O}_{2}$ for 10

327 minutes. RNA Stop Solution (5\% phenol, 95\% ethanol) was added and total RNA was extracted

328 using TRI reagent (Thermo Fisher Scientific). RNA was treated with TURBO DNase (Thermo

329 Fischer Scientific), and was either sent to Yale Center for Genome analysis for RNA sequencing

330 (RNA-seq), or cDNA was synthesized, and quantitative PCR (qRT-PCR) was performed. PCR

331 primers used in this study are listed in Table S7. RNA-seq reads were processed as described

332 previously (59), and deposited in NCBI (PRJNA665492). 


\section{Northern blot}

337 RNA samples were loaded onto either $6 \%$ or $10 \%$ TBE-Urea Gel (Thermo Fischer Scientific)

338 with a biotinylated RNA ladder (Kerafast). Gels were run in 1x TBE buffer at 180V for 60

339 minutes (6\% gels) or $180 \mathrm{~V}$ for 80 minutes (10\% gels). Membranes and filter paper were pre-

340 soaked and RNA was transferred to a Biodyne B Nylon Membrane (Thermo Fischer Scientific)

341 overnight. Membranes were UV-crosslinked using a Stratalinker 2400 UV Crosslinker (1200 mj)

342 and RNA probes (Table S7) were hybridized overnight at $45^{\circ} \mathrm{C}$ with rotation. Hybridization

343 solution was removed and membranes were washed, blocked in Licor Intercept Blocking Buffer

344 and treated with Streptavidin-IRDye $800 \mathrm{CW}$ and examined on a Licor Odyssey scanner.

\section{ACKNOWLEDGEMENTS}

347 We thank Samantha Fancher for assistance with S. marcescens experiments, and Jim Archuleta

348 for bioinformatics support. We are grateful to Boris Görke, Svetlana Durica, Katarzyna Bandyra

349 and Ben Luisi for providing us with RNase E.

\section{FUNDING INFORMATION}

352 This project was supported in part by NIH grants AI133023 and DE028409 to R.R.

354 CONFLICT OF INTEREST

355 The authors have no conflicts of interest to declare. 


\section{REFERENCES}

357 1. Hör J, Matera G, Vogel J, Gottesman S, Storz G. Trans-acting small RNAs and their effects

2. Holmqvist E, Wagner EGH. Impact of bacterial sRNAs in stress responses. Biochem Soc Trans. 2017 Dec 15;45(6):1203-12.

3. Imlay JA. Transcription factors that defend bacteria against reactive oxygen species. Annu Rev Microbiol. 2015 Oct 15;69:93-108. $2008 ; 77: 755-76$. disulfide bond formation. Science. 1998 Mar 13;279(5357):1718-21. RNA represses fhlA translation by blocking ribosome binding. EMBO J. 1998 Oct 15;17(20):6069-75.

371 8. De Lay N, Gottesman S. A complex network of small non-coding RNAs regulate motility in Escherichia coli. Mol Microbiol. 2012 Nov;86(3):524-38. peroxide in Escherichia coli. J Bacteriol. 1999 Jun;181(12):3833-6. 
375 10. Altuvia S, Weinstein-Fischer D, Zhang A, Postow L, Storz G. A small, stable RNA induced

376 by oxidative stress: role as a pleiotropic regulator and antimutator. Cell. 1997 Jul

$377 \quad 11 ; 90(1): 43-53$.

378 11. Zhang A, Altuvia S, Tiwari A, Argaman L, Hengge-Aronis R, Storz G. The OxyS

379 regulatory RNA represses rpoS translation and binds the Hfq (HF-I) protein. EMBO J.

3801998 Oct $15 ; 17(20): 6061-8$.

381 12. Argaman L, Altuvia S. fhlA repression by OxyS RNA: kissing complex formation at two

382 sites results in a stable antisense-target RNA complex. J Mol Biol. 2000 Jul 28;300(5):1101-12.

384

13. Zhang A, Wassarman KM, Ortega J, Steven AC, Storz G. The Sm-like Hfq protein increases OxyS RNA interaction with target mRNAs. Mol Cell. 2002 Jan;9(1):11-22. al. Analysis of pools of targeted Salmonella deletion mutants identifies novel genes affecting fitness during competitive infection in mice. PLoS Pathog. 2009 Jul;5(7):e1000477. the "Enterobacteriales": proposal for Enterobacterales ord. nov. divided into the families Enterobacteriaceae, Erwiniaceae fam. nov., Pectobacteriaceae fam. nov., Yersiniaceae 
fam. nov., Hafniaceae fam. nov., Morganellaceae fam. nov., and Budviciaceae fam. nov.

Int J Syst Evol Microbiol. 2016 Dec;66(12):5575-99.

398

399

400

401

402

403

404

405

406

407

408

409

410

411

412

413

414

415

416

17. Miyakoshi M, Chao Y, Vogel J. Regulatory small RNAs from the 3' regions of bacterial mRNAs. Curr Opin Microbiol. 2015 Apr;24:132-9.

18. Chao Y, Li L, Girodat D, Förstner KU, Said N, Corcoran C, et al. In vivo cleavage map illuminates the central role of RNase $\mathrm{E}$ in coding and non-coding RNA pathways. Mol Cell. 2017 Jan 5;65(1):39-51.

19. Chao Y, Vogel J. A 3' UTR-derived small RNA provides the regulatory noncoding arm of the inner membrane stress response. Mol Cell. 2016 Feb 4;61(3):352-63.

20. Dar D, Sorek R. Bacterial noncoding RNAs excised from within protein-coding transcripts. mBio. 2018 Sep 25;9(5):e01730-18.

21. Chao Y, Papenfort K, Reinhardt R, Sharma CM, Vogel J. An atlas of Hfq-bound transcripts reveals 3' UTRs as a genomic reservoir of regulatory small RNAs. EMBO J. 2012 Oct 17;31(20):4005-19.

22. Hoyos M, Huber M, Förstner KU, Papenfort K. Gene autoregulation by 3' UTR-derived bacterial small RNAs. eLife. 2020 Aug 3;9:e58836.

23. Dutcher HA, Raghavan R. Origin, evolution, and loss of bacterial small RNAs. Microbiol Spectr. 2018 Apr;6(2).

24. Barquist L, Burge SW, Gardner PP. Studying RNA homology and conservation with infernal: from single sequences to RNA families. Curr Protoc Bioinforma. 2016 Jun 20;54:12.13.1-12.13.25. 
417

418

419

420

421

422

423

424

425

426

427

428

429

430

431

432

433

434

435

436

437

25. Lindgreen S, Umu SU, Lai AS-W, Eldai H, Liu W, McGimpsey S, et al. Robust identification of noncoding RNA from transcriptomes requires phylogeneticallyinformed sampling. PLoS Comput Biol. 2014 Oct;10(10):e1003907.

26. Kacharia FR, Millar JA, Raghavan R. Emergence of new sRNAs in enteric bacteria is associated with low expression and rapid evolution. J Mol Evol. 2017 Apr;84(4):204-13.

27. Updegrove TB, Shabalina SA, Storz G. How do base-pairing small RNAs evolve? FEMS Microbiol Rev. 2015 May;39(3):379-91.

28. Chinni SV, Raabe CA, Zakaria R, Randau G, Hoe CH, Zemann A, et al. Experimental identification and characterization of 97 novel npcRNA candidates in Salmonella enterica serovar Typhi. Nucleic Acids Res. 2010 Sep 1;38(17):5893-908.

29. Iyer LM, Koonin EV, Aravind L. Extensive domain shuffling in transcription regulators of DNA viruses and implications for the origin of fungal APSES transcription factors. Genome Biol. 2002 Feb 13;3(3):research0012.1.

30. Hershko-Shalev T, Odenheimer-Bergman A, Elgrably-Weiss M, Ben-Zvi T, Govindarajan S, Seri H, et al. Gifsy-1 prophage IsrK with dual function as small and messenger RNA modulates vital bacterial machineries. PLoS Genet. 2016 Apr 8;12(4):e1005975.

31. Wachter S, Raghavan R, Wachter J, Minnick MF. Identification of novel MITEs (miniature inverted-repeat transposable elements) in Coxiella burnetii: implications for protein and small RNA evolution. BMC Genomics. 2018 Apr 11;19(1):247.

32. Melamed S, Peer A, Faigenbaum-Romm R, Gatt YE, Reiss N, Bar A, et al. Global mapping of small RNA-target interactions in bacteria. Mol Cell. 2016 Sep 1;63(5):884-97. 
438

439

440

441

442

443

444

445

446

447

448

449

450

451

452

453

454

455

456

457

458

33. Raghavan R, Kacharia FR, Millar JA, Sislak CD, Ochman H. Genome rearrangements can make and break small RNA genes. Genome Biol Evol. 2015 Jan 18;7(2):557-66.

34. Gottesman S, Storz G. Bacterial small RNA regulators: versatile roles and rapidly evolving variations. Cold Spring Harb Perspect Biol. 2011 Dec 1;3(12):a003798.

35. Skippington E, Ragan MA. Evolutionary dynamics of small RNAs in 27 Escherichia coli and Shigella genomes. Genome Biol Evol. 2012;4(3):330-45.

36. Peer A, Margalit H. Evolutionary patterns of Escherichia coli small RNAs and their regulatory interactions. RNA N Y N. 2014 Jul;20(7):994-1003.

37. Raghavan R, Groisman EA, Ochman H. Genome-wide detection of novel regulatory RNAs in E. coli. Genome Res. 2011 Sep;21(9):1487-97.

38. Toledano MB, Kullik I, Trinh F, Baird PT, Schneider TD, Storz G. Redox-dependent shift of OxyR-DNA contacts along an extended DNA-binding site: a mechanism for differential promoter selection. Cell. 1994 Sep 9;78(5):897-909.

39. Zheng M, Wang X, Doan B, Lewis KA, Schneider TD, Storz G. Computation-directed identification of OxyR DNA binding sites in Escherichia coli. J Bacteriol. 2001 Aug;183(15):4571-9.

40. Kröger C, Colgan A, Srikumar S, Händler K, Sivasankaran SK, Hammarlöf DL, et al. An infection-relevant transcriptomic compendium for Salmonella enterica Serovar Typhimurium. Cell Host Microbe. 2013 Dec 11;14(6):683-95.

41. Jose BR, Gardner PP, Barquist L. Transcriptional noise and exaptation as sources for bacterial sRNAs. Biochem Soc Trans. 2019 Apr 30;47(2):527-39. 
459

460

461

462

463

464

465

466

467

468

469

470

471

472

473

474

475

476

477

478

479

42. Huber M, Fröhlich KS, Radmer J, Papenfort K. Switching fatty acid metabolism by an RNA-controlled feed forward loop. Proc Natl Acad Sci U S A. 2020 Apr $7 ; 117(14): 8044-54$.

43. Wang C, Chao Y, Matera G, Gao Q, Vogel J. The conserved 3' UTR-derived small RNA NarS mediates mRNA crossregulation during nitrate respiration. Nucleic Acids Res. 2020 Feb 28;48(4):2126-43.

44. Kim HM, Shin J-H, Cho Y-B, Roe J-H. Inverse regulation of Fe- and Ni-containing SOD genes by a Fur family regulator Nur through small RNA processed from 3'UTR of the sodF mRNA. Nucleic Acids Res. 2014 Feb;42(3):2003-14.

45. Mira A, Ochman H, Moran NA. Deletional bias and the evolution of bacterial genomes. Trends Genet TIG. 2001 Oct;17(10):589-96.

46. Williams DL, Slayden RA, Amin A, Martinez AN, Pittman TL, Mira A, et al. Implications of high level pseudogene transcription in Mycobacterium leprae. BMC Genomics. 2009 Aug 25;10:397.

47. Nuss AM, Heroven AK, Waldmann B, Reinkensmeier J, Jarek M, Beckstette M, et al. Transcriptomic profiling of Yersinia pseudotuberculosis reveals reprogramming of the Crp regulon by temperature and uncovers $\mathrm{Crp}$ as a master regulator of small RNAs. PLoS Genet. 2015 Mar;11(3):e1005087.

48. Kalvari I, Argasinska J, Quinones-Olvera N, Nawrocki EP, Rivas E, Eddy SR, et al. Rfam 13.0: shifting to a genome-centric resource for non-coding RNA families. Nucleic Acids Res. 2018 Jan 4;46(D1):D335-42. 
480

481

482

483

484

485

486

487

488

489

490

491

492

493

494

495

496

497

498

499

49. Nawrocki EP, Eddy SR. Infernal 1.1: 100-fold faster RNA homology searches.

Bioinformatics. 2013 Nov 15;29(22):2933-5.

50. Cohen O, Ashkenazy H, Belinky F, Huchon D, Pupko T. GLOOME: gain loss mapping engine. Bioinforma Oxf Engl. 2010 Nov 15;26(22):2914-5.

51. Binns D, Dimmer E, Huntley R, Barrell D, O’Donovan C, Apweiler R. QuickGO: a webbased tool for Gene Ontology searching. Bioinforma Oxf Engl. 2009 Nov $15 ; 25(22): 3045-6$.

52. UniProt: the universal protein knowledgebase. Nucleic Acids Res. 2017 Jan 4;45(Database issue):D158-69.

53. Finn RD, Bateman A, Clements J, Coggill P, Eberhardt RY, Eddy SR, et al. Pfam: the protein families database. Nucleic Acids Res. 2014 Jan;42(Database issue):D222-230.

54. Cock PJA, Antao T, Chang JT, Chapman BA, Cox CJ, Dalke A, et al. Biopython: freely available Python tools for computational molecular biology and bioinformatics. Bioinforma Oxf Engl. 2009 Jun 1;25(11):1422-3.

55. Edgar RC. MUSCLE: multiple sequence alignment with high accuracy and high throughput. Nucleic Acids Res. 2004;32(5):1792-7.

56. Bailey TL, Elkan C. Fitting a mixture model by expectation maximization to discover motifs in biopolymers. Proc Int Conf Intell Syst Mol Biol. 1994;2:28-36.

57. Grant CE, Bailey TL, Noble WS. FIMO: scanning for occurrences of a given motif. Bioinforma Oxf Engl. 2011 Apr 1;27(7):1017-8. 
500 58. Crooks GE, Hon G, Chandonia J-M, Brenner SE. WebLogo: a sequence logo generator. Genome Res. 2004 Jun;14(6):1188-90.

502 59. Wachter S, Hicks LD, Raghavan R, Minnick MF. Novel small RNAs expressed by 


\section{FIGURE LEGENDS}

508 Figure 1. sRNA nodes of origin. sRNAs that arose at each node is depicted by circles. Size and

509 color of each circle corresponds respectively to the number of sRNAs and their ages, as shown in

510 the side panel. Nodes of origin of a few well-studied sRNAs are also marked.

512 Figure 2. sRNAs genes replaced protein-coding genes. Several examples of sRNAs that likely

513 originated from degraded protein-coding genes are shown. (A) oxyS genes in Escherichia coli and

514 Salmonella enterica share sequence homology with the 3' of a peroxidase gene in Serratia

515 proteamaculans. Black arrows represent oxyS and its homologous sequence in peroxidase gene

516 (grey arrow). Peroxidase and $\operatorname{xyS} S$ genes are located divergently from $\operatorname{oxy} R$, and an OxyR-

517 binding site (red box) is present upstream of both $\operatorname{oxy} S$ and peroxidase genes. (B) StyR-3 in

518 Salmonella enterica Typhi and S. enterica Typhimurium share sequence homology with the $5^{\prime}$ end

519 of an MBL-fold metallohydrolase (MMH) gene in Klebsiella pneumoniae. Black arrows represent

520 StyR-3 and its homologous sequence in MMH gene (grey arrow). StyR-3 and MMH genes are

521 located between $\operatorname{ram} R$ and $\operatorname{ram} A$ genes, and a RamR-binding site (blue box) is present upstream

522 of both StyR-3 and MMH genes. (C) STnc240 in Salmonella species share sequence homology

523 with the $3^{\prime}$ end of gabT gene in Cronobacter sakazakii, and both STnc240 and gabT genes are

524 located between yeeY and yoeI genes. (D) STnc3230 in S. enterica Typhimurium and S. enterica

525 Arizonae share sequence homology with the $3^{\prime}$ end of a sugar-binding protein (SBP) gene in

526 Salmonella bongori, and both STnc3230 and SBP genes are located between dapB and carA genes.

527 DapZ, an sRNA transcribed from within $\operatorname{dap} B$, is not shown. (E) IsrK, a prophage-encoded

528 sRNA, shares sequence homology with a region within ASH domains present in several 
529 prophage genes. Black arrows represent is $K$ and its homologous sequences in ASH domains

530 (grey boxes).

531

532 Figure 3. OxyS arose from a peroxidase gene. Arrangement of $0 x y R$, peroxidase and $\arg H$ genes

533 in bacterial families within the order Enterobacterales is shown. In Enterobacteriaceae oxyS

534 (yellow arrow) is found in place of the peroxidase gene (blue arrow). The intergenic region

535 between peroxidase gene and argH varies between families. In members of families

536 Pectobacteriaceae and Yersiniaceae, $3^{\prime}$ ends of peroxidase genes contain OxyS-like sequences

537 (yellow arrows within blue arrows). In Morgenallaceae, transposon-associated genes (white

538 arrows) are located in this region. Orders Pasteurellales and Vibrionales also contain the same

539 gene arrangement as Enterobacterales members. The cladogram is based on Adeolu et al. (16).

540

541 Figure 4. Alignment of E. coli OxyS with 3' end of peroxidase gene in Serratia. The peroxidase

542 gene (blue arrow) in Serratia marcescens ATCC13880 (CP041233) is flanked by oxyR (black

543 arrow) and a dihydrolipoyl dehydrogenase gene (white arrow). ClustalW alignment of oxyS gene

544 in E. coli MG1655 (NC_000913.3) with 3' end of peroxidase gene is shown at the bottom.

545 Nucleotides in blue are part of the peroxidase coding sequence, the stop codon is in red, and the

546 predicted Rho-independent terminator sequence is underlined. The intergenic region between

547 peroxidase and $\operatorname{xyy} R$ genes contains putative OxyR-binding sites (red square).

549 Figure 5. Peroxidase mRNA fragmentation. Short 3' end fragments (marked with *) were 
550 cleaved from peroxidase mRNAs in S. marcescens, E. hoshinae, and $V$. harveyi. “+” indicates

551 samples exposed to $1 \mathrm{mM}$ of $\mathrm{H}_{2} \mathrm{O}_{2}$ for 10 minutes, and non-exposure controls are shown with

552 “-”. Northern blotting was performed with probes that bind to the 3 ' ends of peroxidase

553 mRNAs.

554

555 Figure 6. Two possible routes of OxyS evolution. The 3' end fragment produced from the

556 ancestral peroxidase mRNA was likely non-functional. Top path: In an Enterobacteriaceae

557 ancestor, functional OxyS emerged as a $3^{\prime}$ end-derived sRNA prior to the pseudogenization of

558 the peroxidase gene. Bottom path: OxyS emerged at the $3^{\prime}$ end of an RNA transcribed from a

559 pseudogenized peroxidase gene. Ultimately, the non-functional part of the peroxidase gene was

560 deleted from the genome, resulting in the formation of the oxyS gene. OxyR binds to sites (red

561 boxes) located in the IGR and regulates the expression of peroxidase and oxy $S$ genes. 


\section{Figure 1}

\# sRNAs

sRNA age

old
middle
young
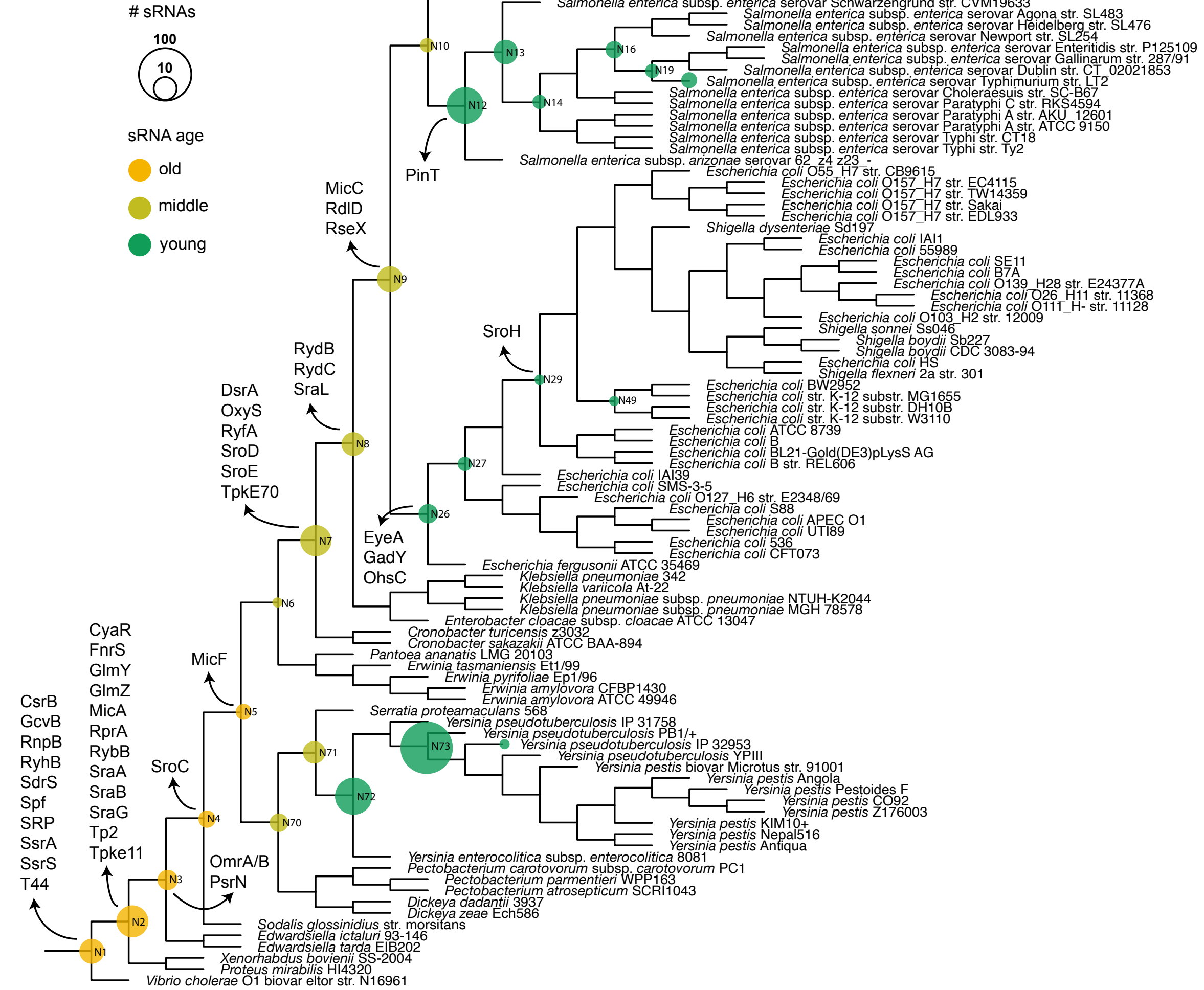


\section{Figure 2}

\section{A. OxyS}

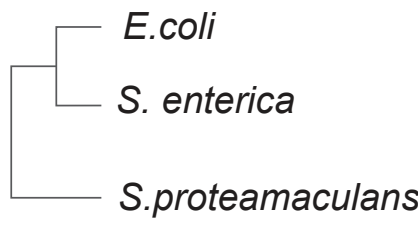

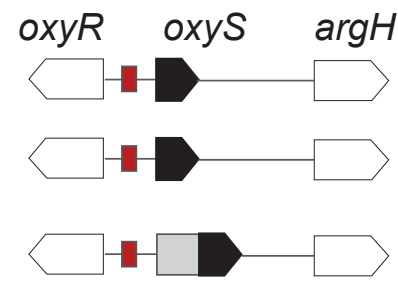

Peroxidase

\section{B. StyR-3}

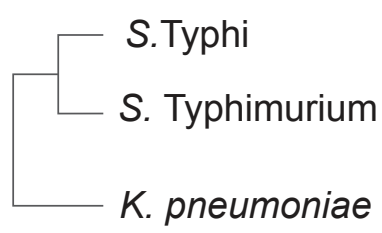

ramR StyR-3 ramA

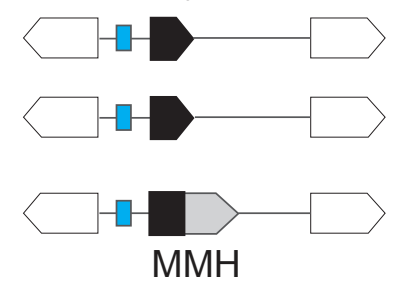

D. STnc3230

C. STnc240

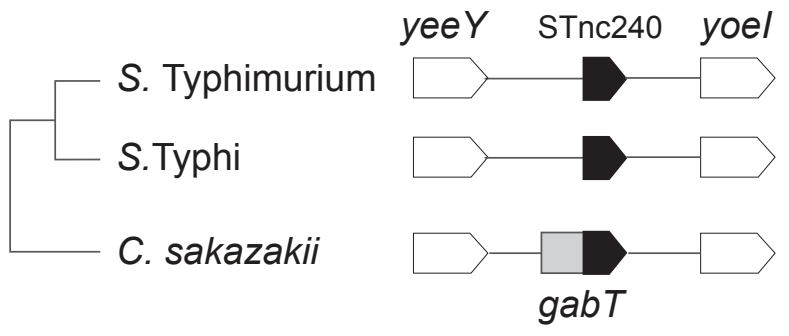

S. Typhimurium

S. Arizonae

S. bongori

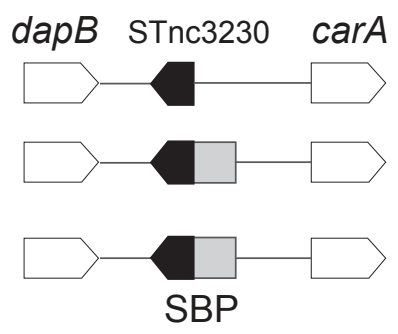

\section{E. IsrK}

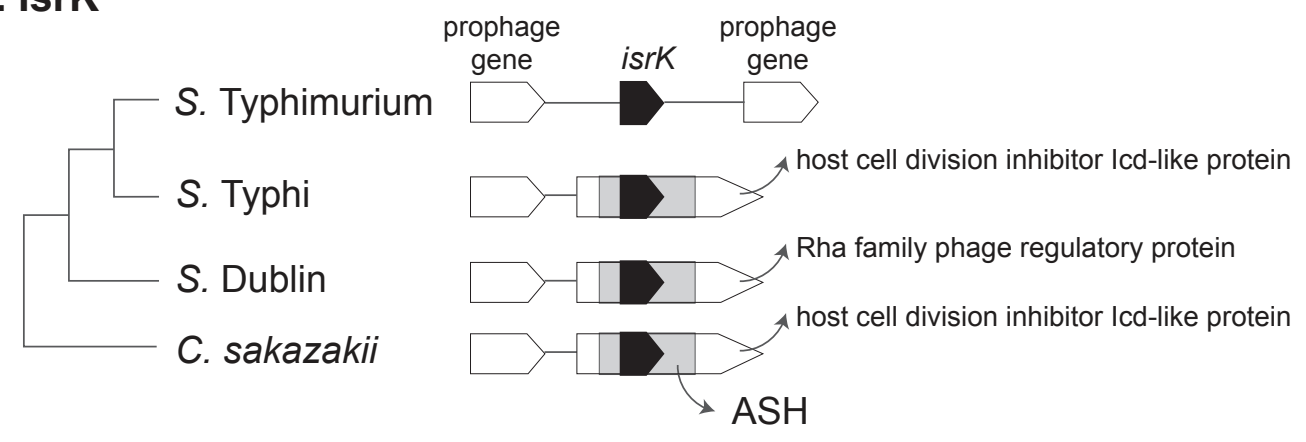


bioRxiv preprint doi: https://doi.org/10.1101/2021.10.18.464853; this version posted October 18, 2021. The copyright holder for this preprint (which was not certified by peer review) is the author/funder, who has granted bioRxiv a license to display the preprint in perpetuity. It is made available under aCC-BY-NC 4.0 International license.

Figure 3

Order

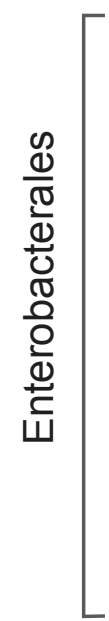

Family

Enterobacteriaceae

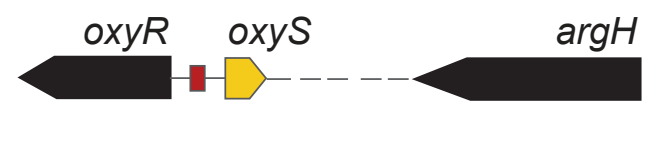

Erwiniaceae

Pectobacteriaceae

Yersiniaceae

Hafniaceae

Morganellaceae

Budiviciaceae

Pasteurellaceae

Vibrionaceae

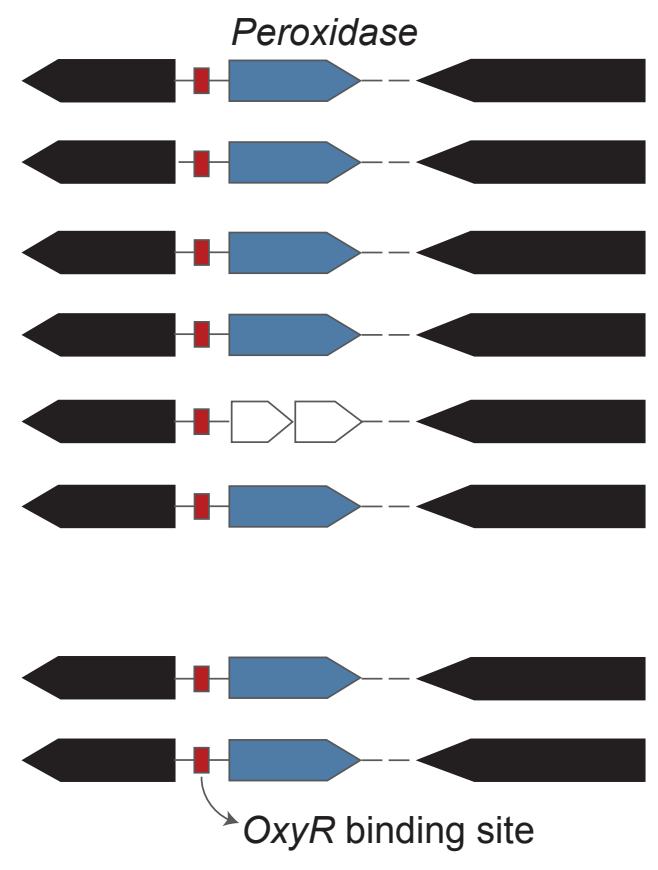


bioRxiv preprint doi: https://doi.org/10.1101/2021.10.18.464853; this version posted October 18, 2021. The copyright holder for this preprint (which was not certified by peer review) is the author/funder, who has granted bioRxiv a license to display the preprint in perpetuity. It is made available under aCC-BY-NC 4.0 International license.

\section{Figure 4}

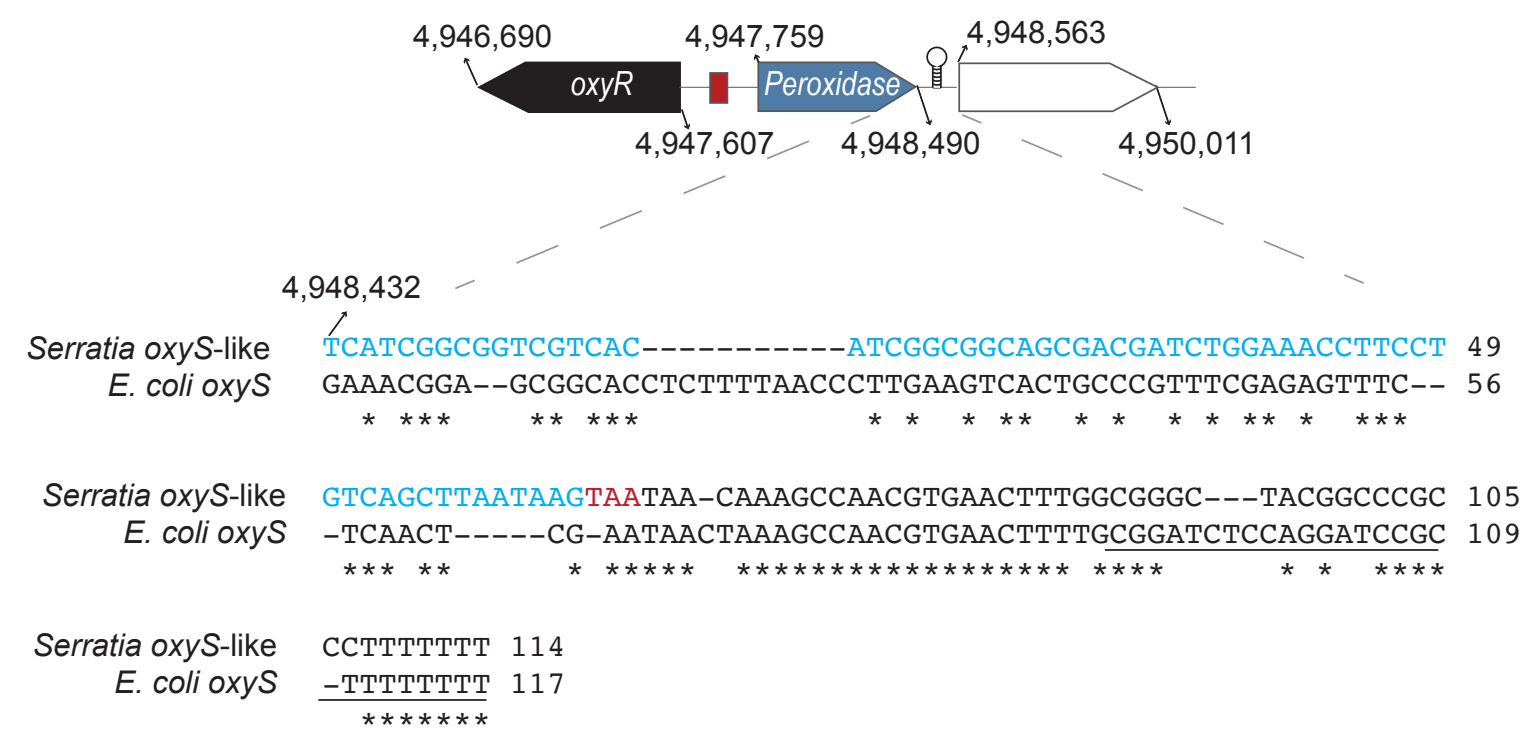




\section{Figure 5}

Serratia

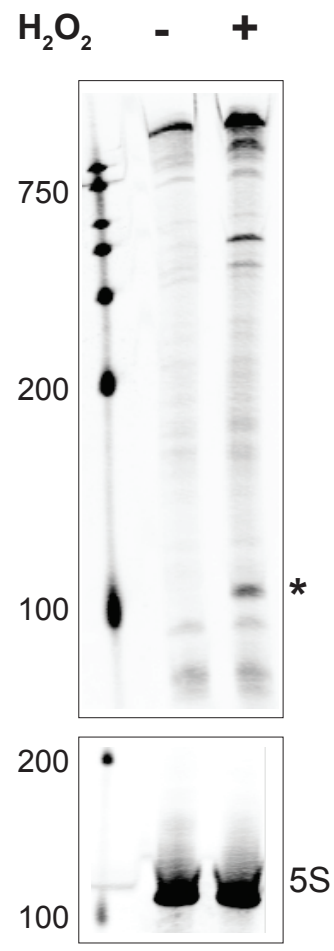

Edwardsiella
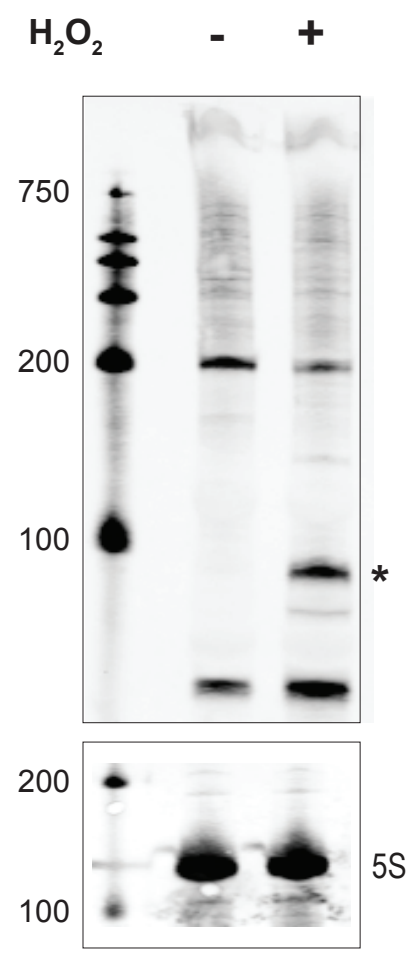

Vibrio
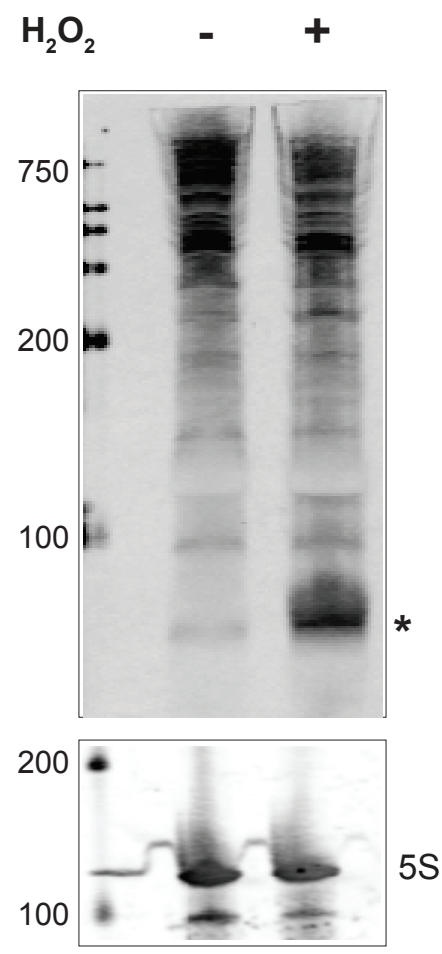
bioRxiv preprint doi: https://doi.org/10.1101/2021.10.18.464853; this version posted October 18,2021 . The copyright holder for this preprint (which was not certified by peer review) is the author/funder, who has granted bioRxiv a license to display the preprint in perpetuity. It is made available under aCC-BY-NC 4.0 International license.

\section{Figure 6}

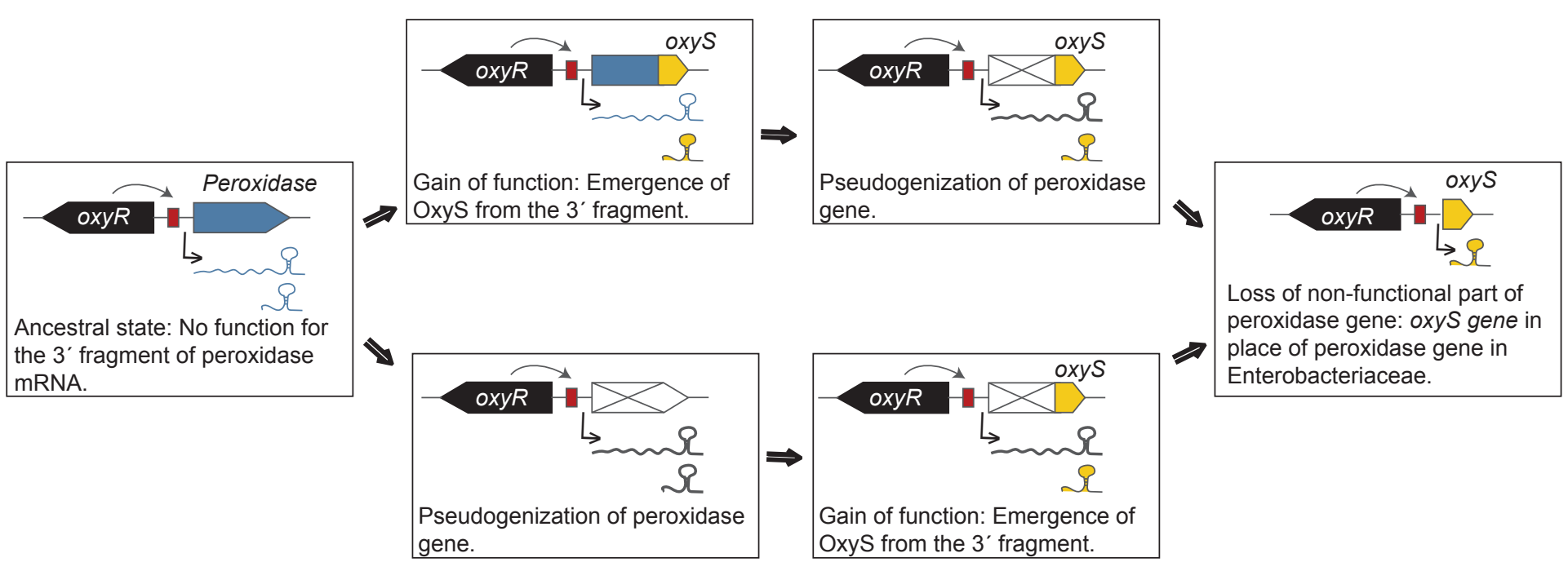

